\title{
Mortalitätsrisiko am Anfang und direkt nach der Behandlung am höchsten
}

Fragestellung: Es werden Ergebnisse von 19 Studien zusammengefasst, um die Mortalität opiatabhängiger Patienten, die mit Methadon oder Buprenorphin substituiert werden, in den verschiedenen Phasen der Substitution abzubilden.

Hintergrund: Die Anzahl opiatabhängiger Patienten ist in den letzten Jahren weltweit stark angestiegen. Eine Substitutionsbehandlung kann mittels Methadon oder Buprenorphin erfolgen und zeigt sich als effektiv um dem Konsum von illegalen Drogen entgegenzuwirken. Beide Substanzen können im Fall einer Überdosis oder eines zusätzlichen Substanzkonsums tödlich sein. Um genaue Einflüsse der Therapiephasen und -dauer auf die Mortalität aufzuzeigen, erfolgte ein Review entsprechender Studien und eine Metaanalyse.

Patienten und Methodik: Nach einer ausführlichen, an den Einschlusskriterien orientierten Literatursuche, wurden 20 Artikel mit 19 Kohortenstudien für die Metaanalyse eingeschlossen. Zunächst wurden die Mortalitätsraten der verschiedenen Gruppen zu den verschiedenen Therapiezeitpunkten unter Berücksichtigung der Todesursache extrahiert. Auf dieser Grundlage wurden anschließend 95\%-Konfidenzintervalle [95\%-KI] berechnet, welche die Mortalitätsrate für die verschiedenen Behandlungszeitpunkte abbilden. Um Vorhersagen über die ersten vier Wochen zu Beginn

Sordo L, Barrio G, Bravo MJ et al. Mortality risk during and after opioid substitution treatment: systematic review and meta-analysis of cohort studies. BMJ 2017; 357: j1550 sowie Beendigung der Behandlung zu treffen, wurden Regressionsmodelle erstellt. Um einen eventuell vorhandenen Bias zu identifizieren, erfolgte die Berechnung des Eggers-Tests.
Ergebnisse: Die generelle Mortalität während der Opiatsubstitution mit Methadon beläuft sich auf 10,6 bis 11,9 Tote von 1.000 Personen und nach Beendigung derselben Substitutionsbehandlung auf 32,5 bis 39,1 Tote pro 1.000 Personen. Bei der Substitution mit Buprenorphin betrug die Mortalitätsrate im Laufe der Behandlung 3,3 bis 5,6 Tote pro 1.000 Personen und nach Beendigung der Behandlung 7,7 bis 12,3 Tote auf 1.000 Personen. An einer Überdosis starben während der Substitution mit Methadon 2,4 bis 2,8 von 1.000 Personen, nach der Methadon-Behandlung 10,6 bis 14,9 pro 1.000 Personen. Im Rahmen einer Buprenorphin-Behandlung starben 1,4 bis 4,6 von 1.000 Personen während oder nach der Behandlung an einer Überdosis. Fasst man die beiden Gruppen zusammen, ist auffällig, dass die generelle Mortalitätsrate unabhängig vom Substitut in den ersten vier Wochen nach Behandlungsende bei 32,1 Toten pro 1.000 Personen liegt und anschließend auf 13,5 Tote pro 1.000 Personen sinkt. Rechnet man die Todesfälle, die durch eine Überdosis bedingt sind, heraus, kommt man zu Beginn der Behandlung auf 3,4 Todesfälle und in den ersten vier Wochen nach einer Methadon-Behandlung auf 4,2 Tote pro 1.000 Patienten. Bei Buprenorphin sind es 10,8 Todefälle vor und 4,2 Tote nach der Behandlung auf 1.000 Patienten, die durch eine Überdosis sterben. Nach den ersten vier Wochen nach dem Behandlungsende sind es noch 3,5 Todesfälle pro 1.000 Patienten, die eine MethadonBehandlung erhalten haben, und an einer Überdosis sterben. Im gleichen Zeitraum sind es 0,9 bis 1,5 Todesfälle auf 1.000 Patienten bei den mit Buprenorphin-Substituierten.

Schlussfolgerungen: Die Mortalität substituierter Patienten ist am Anfang einer Methadon-Behandlung und unmittelbar nach Beendigung einer Substitutionsbehandlung am höchsten.

\section{- Kommentar von Gabriele Koller und Lisa Pröbstl, München}

\section{Todesfälle bei Opioidabhängigkeit vermeiden}

Es handelt sich um eine systematische Übersichtsarbeit und eine Metaanalyse, die sich mit der Mortalitätsrate im Rahmen von Substitutionsbehandlungen opioidabhängiger Patienten beschäftigt. Anhand der dargestellten Daten zeigt sich, dass die Mortalität während einer Substitutionsbehandlung in der Eindosierungsphase von Methadon und in den ersten vier Wochen nach Beendigung einer Substitutionsbehandlung sowohl von Methadon als auch von Buprenorphin besonders hoch ist.

Substituierende Ärzte sollten sich dieser besonderen Risiken bewusst sein und sowohl die Patienten über diese Risiken aufklären, als auch die Patienten in diesen Zeiträumen besonders aufmerksam beobachten und gegebenenfalls die Dosis des Substituts flexibel anpassen beziehungsweise andere Hilfsmöglichkeiten anbieten.

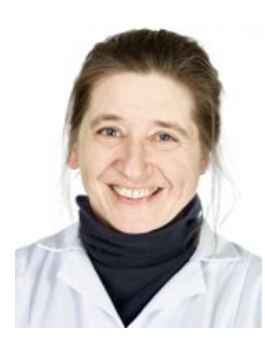

PD Dr. med. Gabriele Koller, München

Oberärztin, Klinik für Psychiatrie und

Psychotherapie, Klinikum der Universität München

E-Mail: Gabi.Koller@med.uni-muenchen.de 